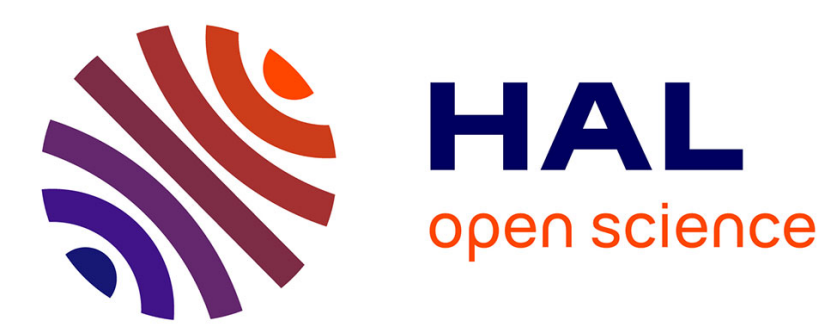

\title{
L'utilisation des micro-drones pour la prospection archéologique à basse altitude
}

Nicolas Poirier, Florent Hautefeuille, Carine Calastrenc

\section{To cite this version:}

Nicolas Poirier, Florent Hautefeuille, Carine Calastrenc. L'utilisation des micro-drones pour la prospection archéologique à basse altitude. Revue Française de Photogrammétrie et de Télédétection, 2017, 213-214 (1), pp.81-94. hal-01892403

\section{HAL Id: hal-01892403 \\ https://hal.science/hal-01892403}

Submitted on 10 Oct 2018

HAL is a multi-disciplinary open access archive for the deposit and dissemination of scientific research documents, whether they are published or not. The documents may come from teaching and research institutions in France or abroad, or from public or private research centers.
L'archive ouverte pluridisciplinaire HAL, est destinée au dépôt et à la diffusion de documents scientifiques de niveau recherche, publiés ou non, émanant des établissements d'enseignement et de recherche français ou étrangers, des laboratoires publics ou privés. 


\title{
L'utilisation des micro-drones pour la prospection archéologique à basse altitude
}

\author{
Nicolas Poirier, Florent Hautefeuille, Carine Calastrenc \\ Équipe Terrae, UMR 5608 TRACES, CNRS - Université Toulouse 2 - Jean-Jaurès, France \\ Maison de la Recherche, 5 Allée Antonio Machado \\ 31058 Toulouse Cedex 9 \\ http://blogs.univ-tlse2.fr/archeodrone/ \\ E-mail : nicolas.poirier1@univ-tlse2.fr, florent.hautefeuille@univ-tlse2.fr, carine.calastrenc@univ-tlse2.fr
}

\begin{abstract}
Résumé
La démocratisation récente des drones engendre une rupture méthodologique en matière de télédétection archéologique. Le premier point concerne la facilité d'utilisation de ces vecteurs, qui fournissent des environnements de vol sécurisés, notamment grâce aux systèmes de navigation GPS et la possibilité de définir des waypoints pour des vols semi-automatisés. Cette flexibilité permet également de répéter des vols sur la même zone. Le deuxième point intéressant pour la télédétection archéologique est la faible altitude de vol des drones, permettant la documentation d'une échelle intermédiaire entre le site et la micro-région. La basse altitude est aussi un moyen d'acquérir des informations de meilleure qualité et de plus grande résolution. Le dernier point concerne le faible coût d'utilisation, même si le prix d'acquisition de l'équipement est assez élevé. Le développement des drones en télédétection archéologique implique donc la définition de nouvelles utilisations pour les capteurs traditionnels. Nous décrivons celles développées dans le cadre du programme toulousain Archéodrones en matière de photogrammétrie et thermographie aéroportée. De nouveaux capteurs seront sans doute adaptés à l'avenir sur drone permettant un accès plus large à ces méthodes de prospection non-invasives.
\end{abstract}

Mots-clés : drone, archéologie, télédétection, thermographie, photogrammétrie

\begin{abstract}
The recent democratization of drones creates a methodological break for archaeological remote sensing. The first point concerns the ease of use of these vectors, which provide secure flight conditions, thanks to the GPS and the ability to set waypoints for semi-automated flight navigation systems. This flexibility also allows repeated flights over the same area. The second interesting point for archaeological remote sensing is the low flight altitude of drones, allowing documentation of an intermediate level between the site and the micro-region. Low altitude is also a way to gain better information and higher resolution. The last point is the low cost of using, even if the purchase price of the equipment is quite high. The development of UAV for archaeological remote sensing implies to define new uses for traditional sensors. We describe those developed in the framework of the Archéodrones program on airborne photogrammetry and thermography. New sensors will probably be adapted on drones in the future, allowing greater access to non-destructive methods of survey.
\end{abstract}

Keywords: UAV, archaeology, remote sensing, thermal survey, photogrammetry

\section{Introduction}

Le développement récent des drones et la miniaturisation des capteurs comme des moyens de transmission de données engendrent une rupture méthodologique en matière de prospection archéologique. En effet, cette démocratisation met à la portée du plus grand nombres la possibilité d'acquérir des données aériennes facilement et à moindre coût. Si cet engouement est aujourd'hui essentiellement limité à des applications de prises de vue illustratives, il faut nous interroger sur les changements de pratiques qui pourront être induits par l'usage de ces nouveaux outils. En effet, la place de la télédétection aérienne dans le champ des méthodes non-invasives d'investigation archéologique n'est peut être pas aussi importante qu'elle le mériterait. Ce désintérêt - ou cette méfiance - à l'égard des informations acquises par télédétection tient sans doute au décalage entre l'échelle d'efficacité des données aériennes (micro-régionale ou régionale selon la résolution spatiale des données) et l'échelle du site à fouiller, ou encore au peu de formalisation des paramètres optimaux de détection des vestiges archéologiques en fonction des conditions 
environnementales ou d'occupation du sol. La place du hasard ou de la chance dans la capacité des données aériennes à révéler la présence de sites enfouis a sans doute conduit à considérer les données de télédétection comme peu fiables pour l'évaluation du potentiel archéologique d'une zone donnée.

Nous essaierons donc de montrer que si les techniques de télédétection appliquées à l'archéologie sont anciennes, l'usage des drones peut conduire à dépasser les limites et inconvénients propres à l'avion, l'hélicoptère ou le satellite pour l'acquisition de données aériennes et favoriser leur utilisation dans une démarche intégrée des méthodes non-invasives et des sondages de diagnostic archéologique.

\section{La télédétection en archéologie}

\subsection{Principes}

La télédétection archéologique vise au repérage de sites archéologiques inédits par l'utilisation de capteurs positionnés à distance de l'objet recherché ou à la collecte d'informations inédites sur des sites déjà repérés par des prospections au sol par exemple. On range la télédétection au rang des méthodes non invasives et non destructives de collecte de données archéologiques, par opposition à la fouille proprement dite qui détruit l'objet de son étude à mesure qu'elle le documente.

La télédétection archéologique est généralement utilisée avant l'organisation d'une fouille ou au départ d'une étude régionale. On la met en œuvre également en amont de grands travaux d'aménagement du territoire (autoroutes, voies ferrées, zones d'activités) pour compléter un état des connaissances acquis par d'autres méthodes sur un espace donné. II s'agit donc d'une étape préalable mais essentielle de la chaîne opératoire du raisonnement archéologique.

La télédétection archéologique repose sur le principe général selon lequel la présence de vestiges enfouis dans le sol modifie ou perturbe la structure du sol. Ces perturbations du sous-sol sont susceptibles d'être détectables à la surface du terrain par des capteurs appropriés dont l'éventail s'étend des simples capacités de l'œil humain jusqu'aux capteurs multispectraux ou micro-topographiques les plus élaborés.

\subsection{Indicateurs et méthodes de détection}

\subsubsection{Indicateurs}

On peut distinguer trois grandes familles d'indices recherchés en surface et pouvant révéler la présence de vestiges archéologiques enfouis. Leur typologie a essentiellement été formalisée dans les années 1970-80 qui ont constitué une forme d'Âge d'Or de la télédétection archéologique, en particulier grâce à l'essor important de la prospection aérienne par avion (Agache, 1978; Bréart et al., 1999).

\section{- Soil marks}

On désigne par "soil marks" des anomalies de couleur du sol détectables en surface, sur sol nu. Ces anomalies peuvent correspondre à des zones où la présence de vestiges enfouis peut entraîner une rétention d'humidité dont témoigne par exemple une couleur plus foncée; Des vestiges enfouis maçonnés peuvent également avoir été remontés jusqu'à la surface par les travaux agricoles récents (en particulier les labours profonds), entraînant la formation d'affleurements pierreux se distinguant de leur environnement par une coloration plus claire du sol (Antrop, 1979; Edis et al., 1989).

\section{- Crop marks}

On désigne par "crop marks" des anomalies visibles dans les champs portant des cultures du fait de la croissance différentielle des plantes qui poussent au-dessus de vestiges enfouis. Les vestiges construits comme les maçonneries ou les sols bétonnés constituent des obstacles au développement racinaire des plantes cultivées. A leur rencontre, les racines souffrent la plupart du temps de stress hydrique qui freine la croissance de la plante par rapport au reste des cultures dont le développement n'est pas entravé. A l'inverse, la croissance des plantes dont le développement s'opère au-dessus d'anciennes structures en creux (fosses, fossés parcellaires par exemple) est favorisée par la rétention d'humidité et le rôle drainant que conservent ces structures, même comblées depuis des siècles. Les plantes présentes au-dessus de ces vestiges connaissent donc un développement accéléré par rapport aux autres. On peut ainsi, en lumière rasante, par le 
jeu des ombres portées, détecter la présence de vestiges enfouis et souvent en restituer l'emprise et le plan, parfois de manière détaillée. II faut ajouter à ces anomalies de croissance les anomalies de couleur des plantes qui témoignent des mêmes conditions de stress ou d'optimum hydrique : les plantes qui poussent au-dessus de vestiges en dur ont tendance à mûrir plus vite que les plantes alentour et donc à prendre une coloration jaunâtre plus tôt, et les plantes qui poussent au-dessus de vestiges en creux connaissent un mûrissement ralenti de sorte qu'elles conservent une couleur verte plus tard que les autres (Figure 1). Ces anomalies de couleur, en plus des anomalies de croissance, peuvent aider à la détection et à la documentation des vestiges enfouis (Aqdus et al., 2012, 2007).

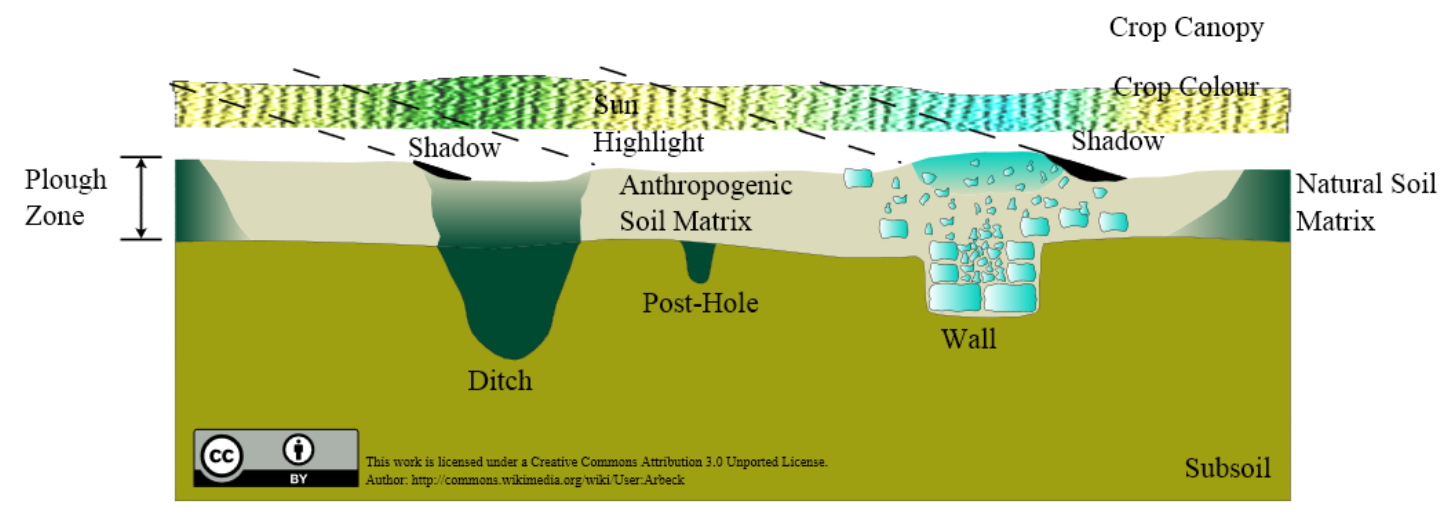

Figure 1 : Principe de formation des cropmarks

\section{- Microreliefs}

Des microreliefs peuvent enfin être détectés à la surface du sol et révéler la présence de vestiges enfouis. C'est particulièrement le cas d'anciens tertres funéraires (tumulus) arasés par le temps et les pratiques agraires répétées, qui peuvent ne se manifester en surface que par la présence de petites éminences difficilement visibles à l'œil nu dans la mesure où elles ne présentent un dénivelé que de quelques centimètres sur des surfaces de plusieurs dizaines ou centaines de mètres carrés. C'est également le cas de certains vestiges de transformation artisanale du minerai de fer qui se manifestent, en plus d'une coloration du sol particulièrement foncée, par la présence de tertres arasés. Les vestiges de structures agraires sont également fréquemment observables sous forme de microreliefs: d'anciennes limites parcellaires fossoyées, même comblées depuis fort longtemps, peuvent encore former de petites dépressions linéaires. A l'inverse, les crêtes de labour, rideaux de cultures ou anciens murs de terrasse peuvent être détectés par la présence de bombements caractéristiques de la surface du sol (Beresford and St Joseph, 1979; Zadora-Rio, 1991). De manière générale, toute anomalie topographique dans le modelé de la surface du sol peut être un indice de la présence d'aménagements anciens ou de vestiges enfouis. Ces microreliefs sont là encore détectables à l'œil nu à la faveur de condition très particulières de lumière rasante.

\subsubsection{Méthodes de détection}

\section{- Photographie aérienne (domaine visible)}

L'emport d'appareils photographiques traditionnels, opérant dans le domaine visible, à bord d'avions est sans doute utilisé en archéologie depuis le début du XXe $s$. Mais son essor est à dater du développement d'une aviation civile amateure, via les aéroclubs, dans les années 1960-1970.

C'est à cette période qu'ont été engagés les premiers programmes de prospection aérienne systématique de territoires relativement vastes, à l'image des prospections pionnières de R. Agache dans le Nord de la France (Agache, 1978).

Ces programmes ont révélé le potentiel archéologique incommensurable des campagnes françaises par l'identification de vestiges enfouis d'habitats, de parcellaire et de voirie révélés par des anomalies de sols et de cultures visibles depuis les airs. Ces découvertes ont démontré 
I'utilité de l'archéologie aérienne pour l'estimation de la richesse archéologique et de la dynamique qui a animé l'occupation du sol en milieu rural, que I'on pensait jusque là largement fossilisée et stable depuis la sédentarisation des sociétés humaines, quasiment sans mouvement entre la clairière de défrichement Néolithique et le village actuel.

\section{- Télédétection satellitaire}

Les années 1970 et suivantes ont vu la démocratisation de l'usage de l'imagerie satellitaire pour la télédétection archéologique, grâce à la déclassification d'images acquises par de grandes agences nationales de recherche, comme la NASA aux États-Unis par exemple.

L'intérêt pour ces images réside essentiellement dans leur caractère généralement multispectral. En plus du domaine visible déjà exploité par la photographie classique, les images satellitaires ont apporté à l'archéologie une information sur les bandes invisibles du spectre lumineux (de l'infrarouge à l'ultraviolet) (Brivio et al., 2000).

La mise en œuvre au profit de l'archéologie d'algorithmes de traitement d'image permettant de combiner plusieurs bandes spectrales a permis d'élargir la gamme des observations de la surface du sol et la détection d'anomalies plus discrètes voire invisibles dans le spectre classique. Ces découvertes ont été favorisées par l'utilisation d'algorithmes de traitement initialement développés pour le monitoring environnemental, comme les index de vigueur de la végétation particulièrement adaptés à l'identification d'anomalies liées au stress hydrique des céréales (Lasaponara and Masini, 2006).

\section{- Scanner laser aéroporté (LiDaR)}

Plus récemment, depuis une vingtaine d'années, la télédétection archéologique de microreliefs, jusque là essentiellement réalisée par photographie aérienne classique grâce à l'observation d'ombres portées en lumière rasante, a été révolutionnée par la technologie du scanner laser aéroporté (Lidar). Le scanner laser, embarqué par avion ou hélicoptère, envoie des impulsions lumineuses vers le sol. Ces faisceaux sont réfléchis par les surfaces rencontrées et enregistrées par le récepteur qui calcule ainsi la distance séparant le point d'émission de la surface rencontrée. Le résultat est un nuage de millions de points en trois dimensions de l'environnement scanné, duquel il est possible d'extraire des modèles numériques de terrain à haute résolution spatiale (centimétrique).

L'intérêt du Lidar en archéologie réside non seulement dans son aptitude à restituer en $3 \mathrm{D}$ de larges étendues à une maille très fine, mais aussi dans la capacité d'une partie des faisceaux lumineux à traverser les branchages des arbres et donc à documenter la topographie des sous-bois forestiers, jusque là invisibles à la prospection aérienne traditionnelle et difficilement lisibles en prospection au sol. L'introduction de cette technique a donc révélé le potentiel archéologique insoupçonné de forêts actuelles, qui apparaissent comme le lieu de fossilisation de structures agraires et paysagères anciennes en grand nombre (Challis et al., 2008; Devereux et al., 2005).

\subsection{Limites}

Avant de décrire les avantages de l'utilisation de microdrones en télédétection archéologique, il paraît utile d'abord de cerner les limites et inconvénients communs aux différentes méthodes d'acquisition de données de télédétection que nous venons de décrire.

Ces méthodes sont d'abord toutes pénalisées par un coût relativement élevé de l'acquisition des données. C'est le cas de la prospection aérienne traditionnelle par avion dont l'heure de vol se situe autour de 150€. C'est également le cas de l'imagerie satellitaire quand on souhaite combiner une forte résolution spectrale à une forte résolution spatiale. Les images acquises par les satellites Ikonos ou Quickbird par exemple peuvent être vendue plusieurs milliers d'euros pour une centaine de kilomètres carrés. Les coûts d'une acquisition de données Lidar enfin, même s'ils sont en forte baisse actuellement, se situent toujours autour de plusieurs dizaines de milliers d'Euros pour des zones de quelques dizaines de kilomètres carrés.

II découle de cette première limite une seconde qui concerne l'impossibilité de répéter les acquisitions de données fréquentes au-dessus du même site ou du même espace. De plus, la nécessaire planification très en amont des missions quelles qu'elles soient (avion, Lidar ou satellite) empêche une bonne maîtrise des paramètres optimaux d'acquisition des données en 
regard des conditions météorologiques ou de couverture végétale du sol, en tous cas à l'échelle fine du site d'intérêt. Si l'archéologie aérienne est un outil très efficace pour la détection de sites inédits ou la documentation plus précise de sites déjà connus ou en cours de fouille, elle reste pénalisée par le peu de formalisation des paramètres d'intervention permettant l'identification de soil marks et crop marks. C'est souvent la chance qui est à l'origine des plus spectaculaires découvertes aériennes, à la faveur de conditions d'éclairage rasants, d'humidité propice ou de mûrissement favorable des cultures, autant de paramètres impossibles à maîtriser quand les missions de survols aériens doivent être planifiées plusieurs semaines à l'avance. II est de plus difficile de répéter fréquemment des missions de survol coûteuses pour observer la variation des résultats en fonction $\mathrm{du}$ changement des paramètres d'intervention.

Enfin, ces différentes méthodes d'acquisition sont généralement caractérisées par la faible résolution spatiale des données acquises (sauf pour le Lidar) du fait de l'altitude de vol des avions utilisés en prospection aérienne qui ne peuvent descendre sous le plancher des $150 \mathrm{~m}$, de la vitesse du vol ou de la faible résolution spatiale des images acquises par satellite. Car si l'imagerie satellitaire a permis d'augmenter la résolution spectrale des observations archéologiques, elle est pénalisée par une résolution spatiale généralement faible des informations acquises. Par exemple, les capteurs Landsat étant les plus complets dans le spectre lumineux livrent des images dont la résolution spatiale du pixel n'est que de 15 ou 30 $\mathrm{m}$ au sol, ce qui peut être notoirement insuffisant pour la télédétection de vestiges archéologiques peu étendus.

\section{Les principaux avantages des micro-drones en archéologie et les changements de pratiques induits.}

\subsection{Faible coût d'exploitation}

Le premier intérêt de l'usage des micro-drones en télédétection archéologique est leur faible coût d'exploitation comparativement aux autres méthodes décrites. Si l'investissement de départ est assez conséquent (plusieurs dizaines de milliers d'Euros pour une plateforme associée à une station-sol), la rentabilité est accrue par la possibilité de répéter de nombreux vols avec un coût d'exploitation limité à quelques centaines d'Euros par an pour le remplacement des consommables que sont les batteries Lithium ou autre petit matériel.

\subsection{Abaissement des altitudes de vol pour un gain de résolution et la documentation d'une échelle spatiale intermédiaire entre le site et la région}

Le principal intérêt des micro-drones réside naturellement dans l'abaissement des altitudes de vol par rapport aux vecteurs traditionnels que sont I'avion ou l'hélicoptère. Ces derniers sont limités à un plancher situé à $150 \mathrm{~m}$ au-dessus du sol qui constitue à l'inverse le plafond de l'usage des micro-drones. Cet abaissement engendre mécaniquement un gain de résolution spatiale des images acquises qui sont souvent plus adaptées à la documentation à l'échelle d'un site. II est également intéressant de pouvoir faire varier ces altitudes de vol sur toute la gamme possible entre $10 \mathrm{~m}$ et $150 \mathrm{~m}$ dans le cadre d'un vol stationnaire pour produire des images différentes du même espace (Figure 2). Le micro-drone apparaît ainsi comme un outil adapté à la documentation d'une échelle intermédiaire entre celle du site (qui pouvait être documenté auparavant par l'usage de ballons, de cerf-volants ou de perches télescopiques) et l'échelle micro-régionale qui peut être traitée par avion. Le micro-drone permet une investigation à l'échelle de la parcelle, du groupe de parcelles ou du petit terroir, ce qui est particulièrement utile par exemple dans un contexte d'archéologie préventive souvent amenée à intervenir en amont d'aménagements qui correspondent à cette échelle (lotissements, ZAC, etc.). 

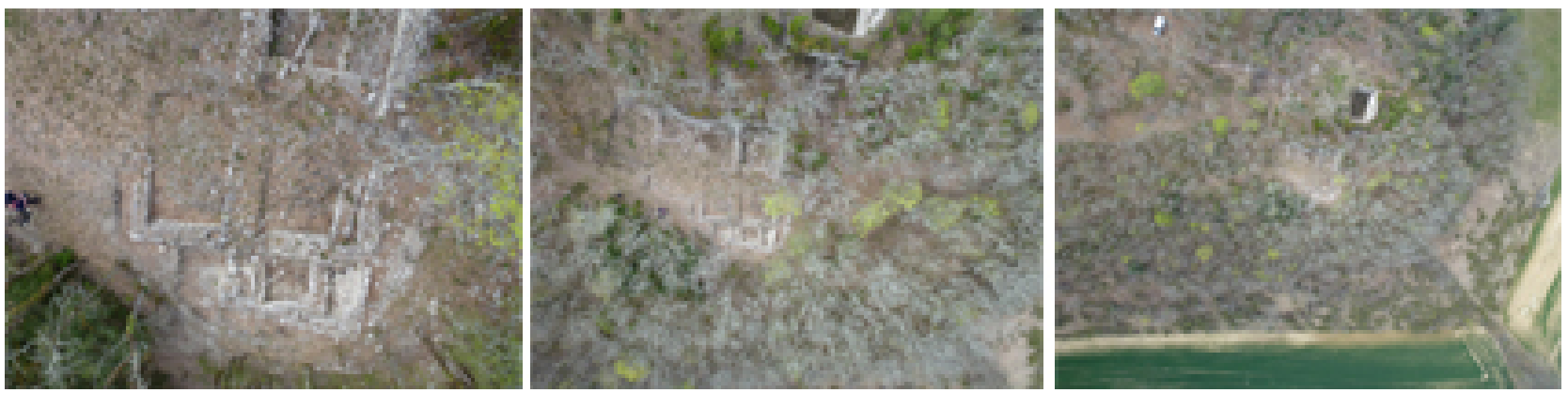

Figure 2 : Exemples de prises de vue du même site à des altitudes différentes $(10 \mathrm{~m}$ à gauche ; $50 \mathrm{~m}$ au centre ; $150 \mathrm{~m}$ à droite)

\subsection{Flexibilité d'utilisation et environnement de vol sécurisé}

Un intérêt important de l'usage des micro-drones en télédétection archéologique réside dans leur environnement de vol aujourd'hui sécurisé qui permet leur prise en main par des non-spécialistes moyennant une solide formation initiale théorique et pratique. En effet, les plateformes actuellement commercialisées sont équipés de stabilisateurs inertiels basés sur les informations fournies par des accéléromètres et gyroscopes embarqués, permettant une très bonne stabilité du vol sans action directe du télé-pilote. De même, l'équipement des plateformes en GPS permet, en liaison avec une station au sol, de programmer des plans de vols semi-automatisés sur la zone d'intérêt à survoler. Le nombre de points de passage, leur équidistance, leur altitude et le temps d'arrêt sur chacun d'eux peuvent être planifiés à l'avance afin de documenter la zone d'intérêt avec la précision souhaitée (Figure 3).

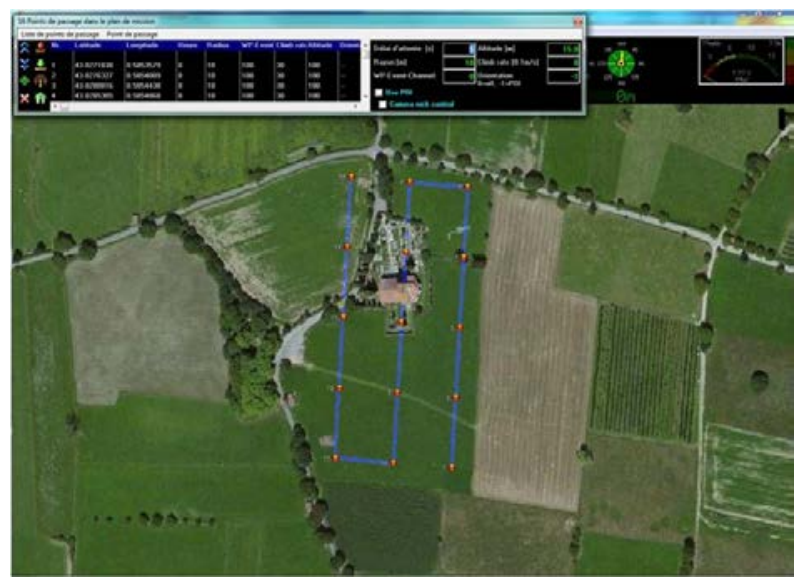

Figure 3 : Définition de waypoints pour la couverture systématique et automatisée de parcelles.
De même, le faible encombrement du matériel nécessaire associé à cette facilité de mise en œuvre permet une prise de décision rapide pour la réalisation des survols, en fonction des conditions météorologiques du moment. Selon ces paramètres, il peut être décidé de réaliser un survol du jour au lendemain, voire même du matin pour l'après-midi. L'ensemble du matériel peut être embarqué dans un utilitaire de faible volume ou dans le coffre d'une berline et transporté sur le lieu de vol.

Cette souplesse d'utilisation est très précieuse en télédétection archéologique car elle permet de choisir les paramètres optimaux de réalisation du vol en fonction des vestiges recherchés (conditions atmosphériques et humidité du sol par exemple). Elle permet surtout de faire varier ces paramètres par des survols répétés au-dessus de la même zone d'intérêt afin de maximiser les chances de détection de vestiges. En effet, on sait que des conditions de vol données ne seront pas favorables à la détection de tous les types de vestiges, les maçonneries enfouies étant révélées dans des contextes différents de ceux favorables à l'identification de structures en creux. Le diagnostic complet d'une zone donnée ne peut donc être réalisé de façon fiable que par des survols répétés à différents moment de la saison culturale, ce que permet la souplesse de mise en œuvre des micro-drones. On peut ainsi compter de façon plus assurée sur la maîtrise des paramètres de détection des vestiges que sur le "facteur chance" qui est le plus souvent à l'œuvre dans la télédétection aéroportée ou satellitaire.

\section{Nouveaux usages d'anciens capteurs et perspectives de développement de nouveaux capteurs}

Le développement récent de l'usage des drones 
en archéologie procède d'une miniaturisation des système de navigation qui a rendu leur pilotage plus sûr et plus facile même à des non spécialistes, et celle des capteurs généralement utilisés en télédétection, ce qui rend le couplage des deux beaucoup plus efficace.

\subsection{Thermographie à basse altitude}

La miniaturisation récente des capteurs infrarouges ou multispectraux désormais disponibles sous forme de petits appareils portables légers permet d'envisager leur emport sur des vecteurs aériens de type micro-drones. On comprend aisément que ce couplage permet de pallier les limites de la télédétection multispectrale traditionnellement réalisée à partir d'images satellites ou de survols par avion ou hélicoptère pour ce qui concerne la seule prospection thermique aéroportée. La capacité de vol à basse altitude permet d'associer une fine résolution spatiale à une fine résolution spectrale. Dans le cadre du programme Archéodrones développé au sein de la plateforme Terrae de I'UMR 5608 TRACES (CNRS - Université Toulouse 2 Jean-Jaurès), nous testons le couplage d'un microdrone octorotor de type Mikrokopter Okto XL avec une caméra thermique de type FLIR T620 pour la télédétection de vestiges archéologiques (Poirier et al., 2013). Cette caméra permet des prises de clichés thermiques situés dans la gamme de l'infrarouge thermique (7.5 à $14 \mu \mathrm{m})$, avec une sensibilité de $0.04{ }^{\circ} \mathrm{C}$. La résolution d'image est relativement faible ( $480 \times 640$ pixels), mais peut toutefois être compensée par l'abaissement des altitudes de vol. Elle pèse 1.3 $\mathrm{kg}$, ce qui permet de l'embarquer dans la nacelle du microdrone MK Okto XL

L'objectif est d'identifier des anomalies de rayonnement infrarouge thermique mesuré à la surface du sol pouvant être interprétées comme révélatrices de la présence de vestiges enfouis. Le repérage de ces anomalies répond à des logiques différentes selon que le terrain survolé est nu ou porte des cultures.
Sur sol nu, on cherche à mesurer des anomalies lisibles à la surface du sol et produites par l'inertie thermique des vestiges enfouis. Le principe est que ces vestiges réagissent aux changements de température du sol à une vitesse différente du sédiment encaissant. Dans le cas de structures maçonnées, l'inertie thermique sera plus grande que le sédiment meuble entourant les vestiges, et inversement dans le cas de structures fossoyées. Cette différence d'inertie thermique provoque une anomalie de température en sous-sol transmise par rémanence jusqu'à la surface où elle peut être repérée par la caméra infrarouge.

Sur un terrain portant des cultures, Les mesures prises ne concernent pas la surface du sol (invisible) mais plutôt les plantes cultivées elles-mêmes. Les anomalies recherchées sont alors liées à la vigueur du développement de celles-ci, conditionnée par leur niveau de stress hydrique. Comme explicité rapidement plus haut, si le développement racinaire d'une plante cultivée est entravé par la présence de maçonneries enfouies, la plante est privée d'une partie des apports en eau et en nutriments qui lui sont nécessaires, relativement aux autres plantes voisines qui ne se développent pas dans le même contexte. A l'inverse, le développement racinaire peut être favorisé si la plante pousse au-dessus d'anciennes structures en creux dont le comblement, différent par sa structure du substrat environnant, favorisera la rétention de l'humidité et donc un mûrissement ralenti. Alors que ces indices étaient identifiables auparavant uniquement dans le domaine visible, par photographie classique, essentiellement pendant un laps de temps réduit de la saison culturale correspondant à l'épiaison et au début de mûrissement, les différences de stress hydrique des plantes cultivées sont lisibles bien plus tôt dans la saison alors même que rien n'apparaît en photographie classique. La délimitation des anomalies est de plus généralement affinée par imagerie infrarouge en comparaison du domaine visible (Figure 4 et Figure 5). 


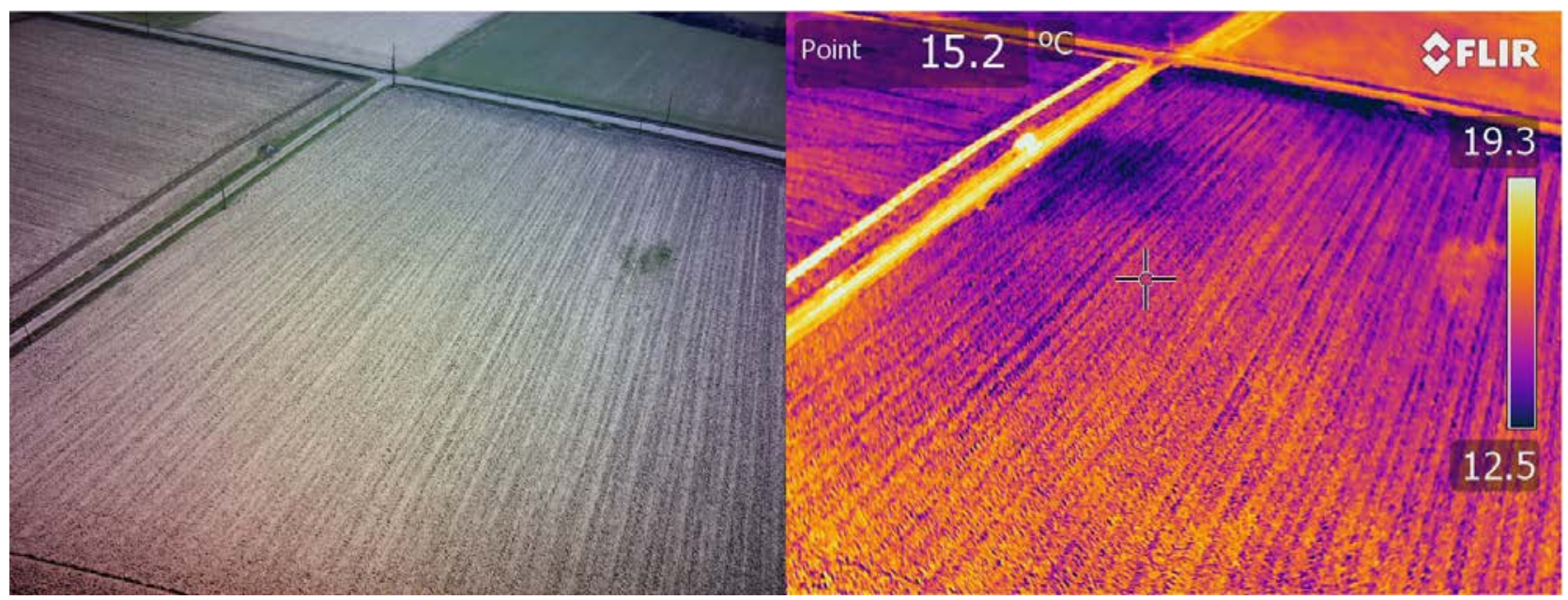

Figure 4 : Thermographie sur sol nu à Saint-Porquier (82). Aucune anomalie n'est visible sur la photographie classique (à gauche) alors qu'une anomalie froide est visible dans le coin supérieur gauche de la parcelle (à droite) correspondant à l'emprise d'un site archéologique repéré en prospection au sol.
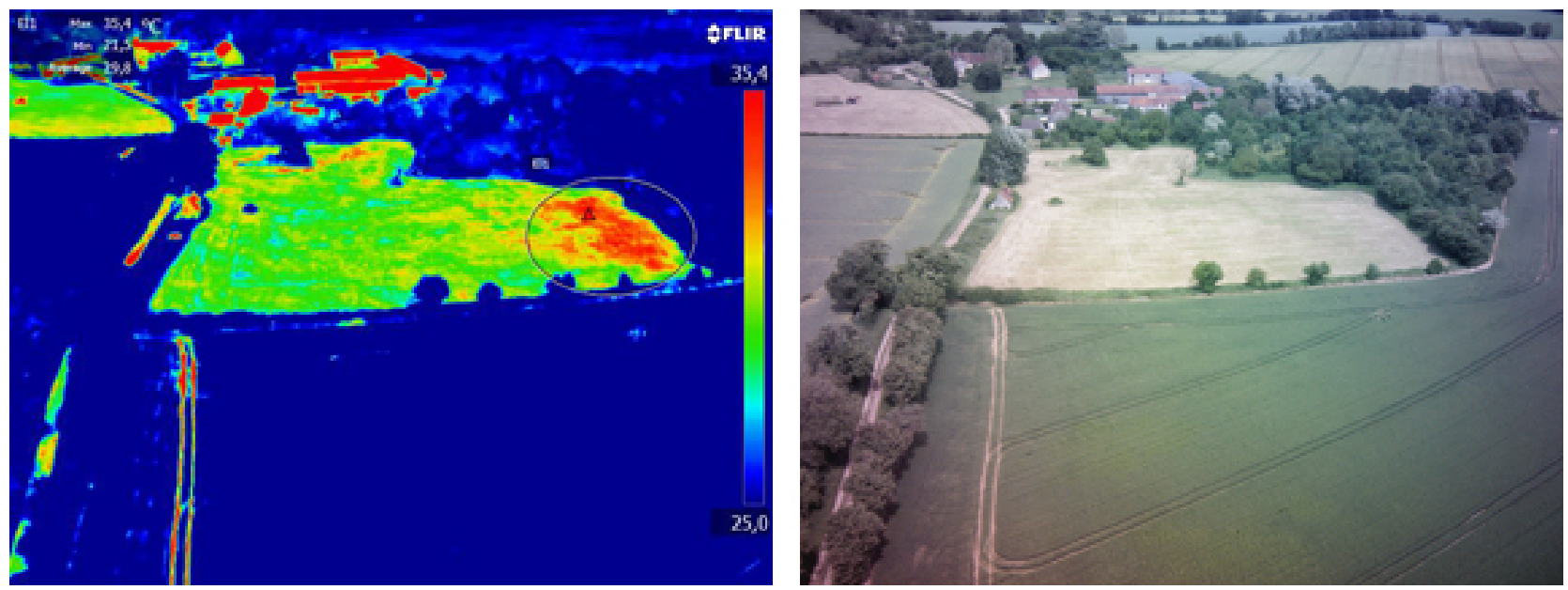

Figure 5 : Thermographie sur couverture végétale à Sancergues (18). Une anomalie chaude est clairement délimitable au sein d'une parcelle de température homogène (à gauche), à l'emplacement d'un indice de site gallo-romain identifié par des ramassages de surface. Aucune anomalie n'est visible sur le cliché classique (à droite).

L'abaissement de l'altitude de vol permet désormais d'envisager le repérage d'anomalies correspondant à de petits bâtiments, alors même que la prospection thermique aéroportée par avion avait jusqu'à présent permis la détection surtout de macrostructures paysagères comme des voies ou des limites parcellaires fossiles. Ajoutons que la souplesse d'utilisation du micro-drone permet la détection et le suivi d'une anomalie sur l'ensemble d'une saison culturale. Les premiers tests réalisés sur un corpus de sites témoins ont déjà permis de constater que, par exemple, la recherche d'anomalies sur sol nu était favorisée par des contextes de vol au lever du jour dans les saisons de forte amplitude thermique journalière; au contraire, la recherche d'anomalies liées au stress hydrique des plantes est favorisée lors de vols menés en fin de journées chaudes.

La disponibilité récente de caméras multi- ou hyper- spectrales au même format portable permet même d'envisager l'adaptation complète des procédés de télédétection satellitaire à des survols à basse altitude. II s'agit donc d'une voie de renouveau possible pour cette méthode de télédétection archéologique jusque là pénalisée par sa faible résolution spatiale.

\subsection{De la photographie à la photogrammétrie}

Les micro-drones sont essentiellement utilisés en 
archéologie aujourd'hui pour l'emport d'appareils photographiques numériques classiques, dans le but de réaliser des clichés de sites fouillés ou en cours de fouille, dans un but essentiellement illustratif, de valorisation grand public ou pour acquérir une documentation orthophotographique utile à la production de plans géoréférencés. Mais une application utile du couplage d'un micro-drone avec un appareil photographique classique peut aussi concerner l'acquisition de données sur des édifices ou des parties d'édifices particulièrement difficiles d'accès (comme des façades de clochers d'églises ou de tours de châteaux par exemple. De même, un nombre plus réduit d'équipes travaillent à l'utilisation du couplage d'un micro-drone avec un appareil photographique classique pour la réalisation de relevés photogrammétriques.

En effet, les progrès en matière de résolution des images numériques et la possibilité de les acquérir en grand nombre, avec un fort taux de recouvrement, grâce à l'utilisation d'un micro-drone, permet de couvrir des zones de plusieurs hectares en vue de la modélisation 3D photogrammétrique utile à la détection des micro-reliefs qui pourraient s'y trouver.

De plus, l'augmentation constante des capacités de calculs des ordinateurs accessibles au plus grand nombre, et la mise à disposition d'algorithmes permettant l'automatisation d'un certain nombre d'étapes de la restitution 3D photogrammétrique (appariement des images, mosaïquage par exemple) rend la méthodologie accessible à des non-spécialistes, en apparence au moins.

Il apparaît donc utile au travail de l'archéologue de réaliser des restitutions 3D photogrammétriques pour la détection de micro-reliefs, pour l'étude d'édifice encore en élévation ou pour la restitution de contextes difficiles d'accès aux méthodes de relevés plus conventionnelles (comme les secteurs de falaises aménagées). Nous ne développerons pas l'intérêt évident de produire ce genre de restitutions à des fins de valorisation auprès du grand public ou de communication, pour nous concentrer sur les apport scientifiques de cette approche. Par exemple, la restitution 3D par photogrammétrie permet un gain de temps non négligeable dans la démarche de relevé de micro-reliefs par comparaisons aux techniques plus traditionnelles qui consistent en un maillage de points relevés à l'aide de GPS différentiels ou d'une station totale. La surface de la zone à traiter et la finesse du maillage souhaité peuvent conduire à des temps de travail sur le terrain pouvant atteindre plusieurs heures, voire plusieurs jours. L'usage d'un micro-drone équipé d'un appareil photographique numérique permet de couvrir une surface d'environ 4 à 5 ha au cours d'un vol d'une dizaine de minutes. La variation des altitudes de vol peut être ajustée en fonction de la discrétion des micro-reliefs à détecter.

Cette démarche a été mise en œuvre dans le cadre du programme Archéodrones sur le site de Thémis (Pyrénées-Orientales), où les résultats de la détection de micro-reliefs par photogrammétrie ont pu ensuite être confrontés à des prises de mesures sur le terrain puis à des sondages archéologiques mécaniques (Figure 6). De même, la procédure a été testée sur le site de la Silla del Papa (Espagne) pour documenter en 3D des aménagements protohistoriques réalisés sur des falaises particulièrement difficiles d'accès (Figure 7) ou encore pour restituer en trois dimensions le village médiéval de Flaugnac (Lot) (Figure 8). 


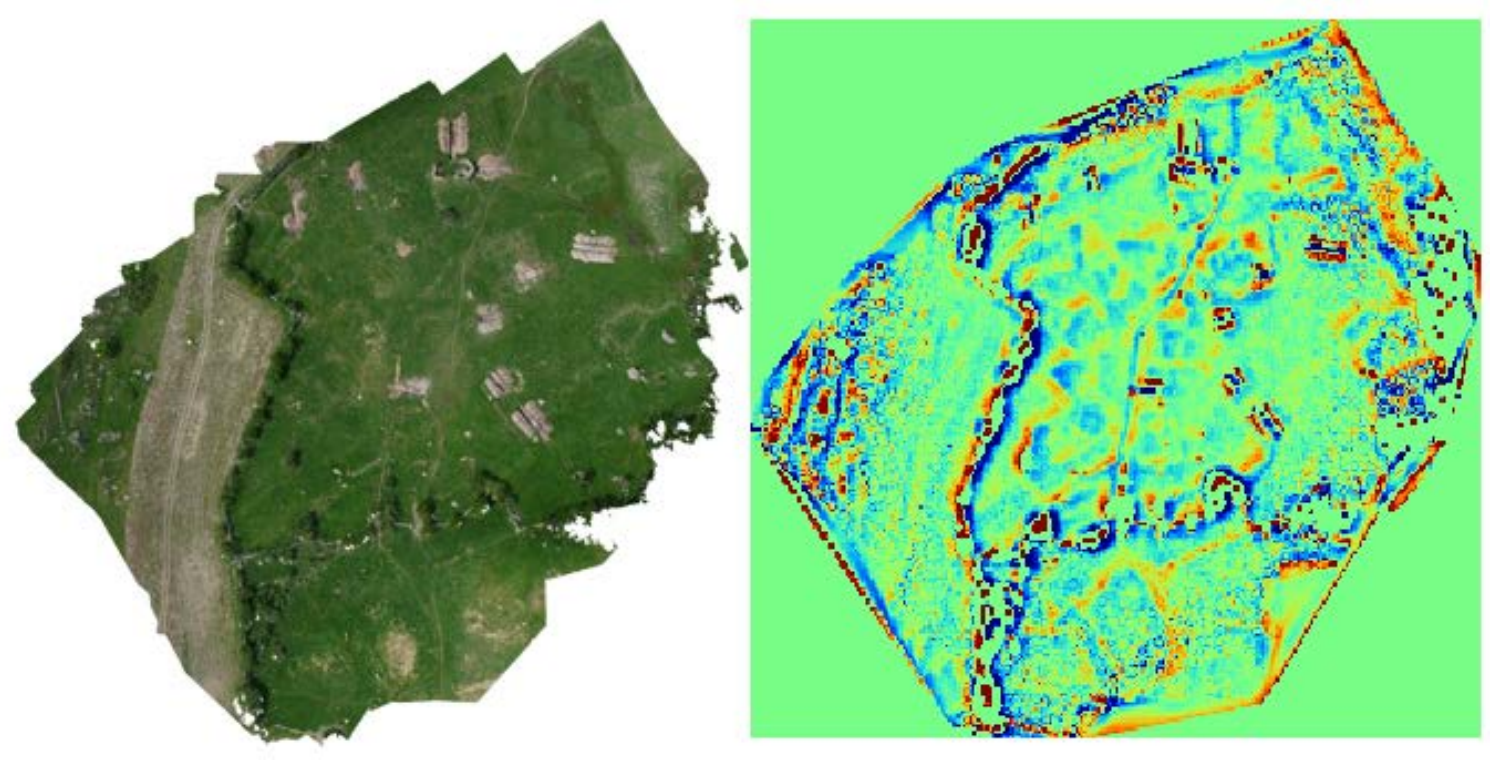

Figure 6 : Un relevé photogrammétrique par drone sur le site de Thémis a permis de cartographier les microreliefs agraires. A gauche, orthophotographie du site ; A droite, cartographie des micro-reliefs.
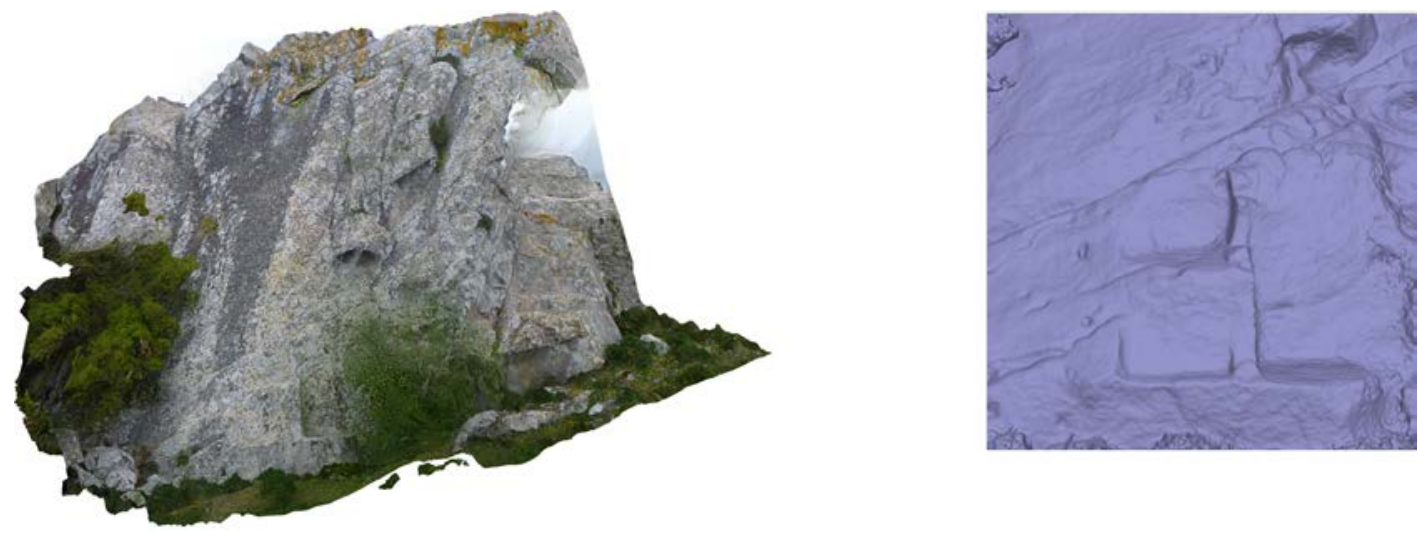

Figure 7 : La couverture photogrammétrique d'une falaise de la Silla del Papa, Espagne (à gauche) a permis la génération d'un modèle $3 D$ où sont clairement visibles les aménagements anthropiques réalisés sur cette falaise pour l'installation de bâtiments (à droite). 

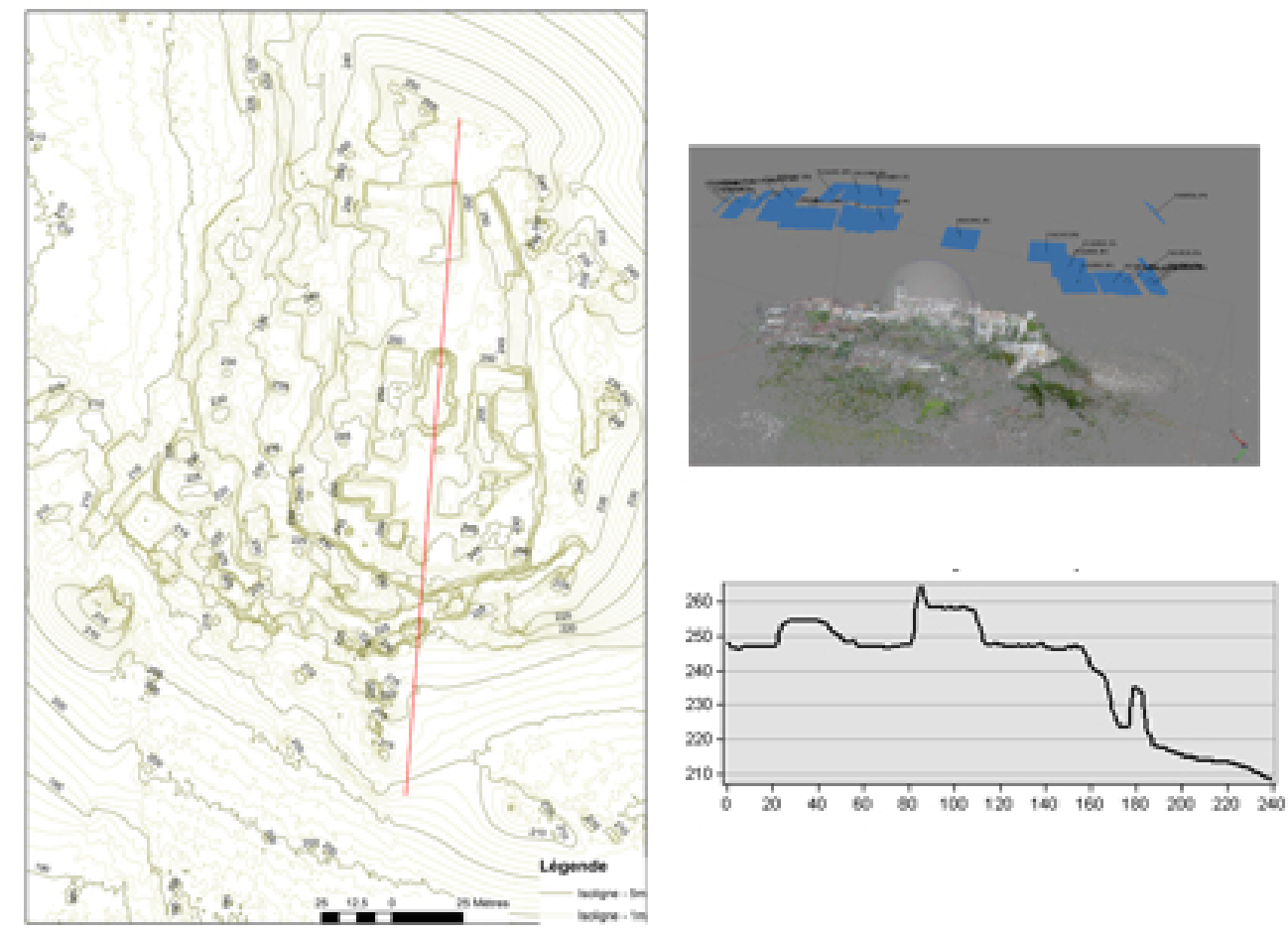

Figure 8 : La couverture photographique par drone du village de Flaugnac (Lot) a permis de générer un modèle 3D à l'échelle du site et de restituer le village dans son contexte topographique local.

On envisage également de réaliser des modelés numériques de surface des plantes cultivées, à différents moments de la saison culturale, non seulement pour repérer des anomalies de croissance à un instant $t$, mais aussi pour mesurer ces retards de croissance par le croisements des différents modelés, et ainsi ouvrir la voie d'une modélisation temporelle des anomalies de culture, comme c'est déjà le cas en monitoring agricole.

L'usage de la photogrammétrie par drone permet donc un gain de temps non négligeable sur le terrain, qui est souvent l'étape la plus onéreuse. Mais elle permet aussi la production de données qui seraient parfois impossibles à acquérir avec des moyens conventionnels.

\subsection{Perspectives de nouveaux capteurs embarqués par drone}

Nous avons décrit les avantages de l'utilisation des drones pour les applications que nous développons actuellement en matière de photographie classique, de thermographie et de photogrammétrie, mais il est certain que d'autres capteurs pourront être adaptés à une utilisation archéologique embarquée par drone. Les scanner laser aéroportés par exemple (LIDAR) pourraient utilement être embarqués par drone, de manière à accroître la densité des points au sol, ou pour scanner des édifices d'intérêt, de manière complémentaire à l'utilisation des scanners terrestres. Des expériences prometteuses ont déjà eu lieu en ce sens. Leur généralisation, et la mise à disposition de solutions clés en main, permettraient un accès facilité au scanner laser aéroporté qui demeure une technologie coûteuse, surtout quand les zones d'intérêt sont peu étendues.

Il est certain également que d'autres méthodes de prospection archéologiques, généralement réalisées au sol, pourraient gagner en productivité par une adaptation sur drone aérien. On pense particulièrement aux méthodes de prospection magnétiques et électro-magnétiques, qui sont opérées depuis le sol mais sans contact direct avec celui-ci, si ce n'est à une distance réduite. Leur utilisation par des moyens pédestres ou faiblement mécanisés contraint leur utilisation à quelques hectares par jour. Une adaptation par drone pourrait conduire à accroître très fortement ce rayon d'action. Mais cette adaptation soulève des questions techniques difficiles qui tiennent dans la possibilité de réaliser des survols à l'échelle parcellaire à des altitudes constantes très 
basses et à vitesse relativement réduite. De plus, les capteurs demandent à être isolés au maximum de toute perturbation électro-magnétique, donc probablement du vecteur lui-même. Un certain nombre de verrous techniques freinent encore cette adaptation qui serait très profitable au développement et à la généralisation de ces méthodes de prospections, notamment dans le cadre de l'archéologie préventive.

Enfin, les progrès récents réalisés en matière de vol autonome seraient profitables à l'archéologie pour l'exploration de grottes et autres cavités souterraines inaccessibles à l'Homme, soit pour des raisons de sécurité (environnement toxique dans le cas d'anciennes zones de mines par exemple), soit à cause du risque d'endommager d'éventuels vestiges ou sols archéologiques encore en place. Là encore, les verrous techniques sont importants. Ils concernent le rapport taille/emport pour un appareil devant évoluer dans des diverticules exigus; les capacités de géolocalisation du drone en milieu confiné, hors signal GPS; les moyens de transmissions de données pour une cartographie 3D en temps réel de la cavité explorée ; et surtout le défi de l'autonomie énergétique nécessaire à une exploration longue (plusieurs dizaines de minutes). Mais nous ne doutons pas que ces verrous pourront être levés si l'on considère l'intérêt stratégique d'autres applications possibles de ce genre d'appareil (protection civile pour la recherche de personnes disparues lors d'effondrements de bâtiments, anti-terrorisme, etc.).

\section{Conclusion}

Pour conclure, nous rappellerons en guise de synthèse les principaux avantages de l'utilisation des microdrones en télédétection archéologique. L'abaissement des altitudes de vol permet un gain de résolution spatiale des données acquises avec des capteurs généralement embarqués par avion, hélicoptère ou même satellites. Leur souplesse d'utilisation permet de réaliser des survols répétés de la même zone d'intérêt à de très faibles intervalles de temps, de manière à acquérir de l'information dans des contextes météorologiques et de couvertures végétales variées. Cela est également possible par le faible coût d'exploitation des microdrones même si l'investissement de départ peut paraître élevé pour des projets de recherche fondamentale publique.

En somme, I'utilisation des microdrones en télédétection archéologique constitue une évolution importante dans la mesure où elle met l'approche aérienne à la disposition d'un plus grand nombre d'utilisateurs. Nous espérons que leur usage ne tardera pas à se généraliser et que cette évolution permettra de redonner aux méthodes de prospections non-invasives (Trément, 2000) toute la place qu'elles méritent dans la démarche d'évaluation du potentiel archéologique, notamment en contexte préventif, aux côtés des méthodes de sondages mécaniques qui restent indispensables. En effet, l'établissement d'un référentiel permettant d'optimiser la détection de traces archéologiques dans le sol devrait permettre de fournir une information sur la densité des vestiges présents sur un secteur donné, et ainsi contribuer à la définition d'une stratégie efficace de diagnostic archéologique mécanisé, en concentrant les moyens d'abord sur les zones de plus fort potentiel. En contexte de recherche programmé, de la même manière, la multiplication des images d'un même site, par des capteurs et dans des conditions d'intervention variés, fournira une image plus complète des structures enfouies et de là, une estimation plus juste des moyens à mettre en œuvre pour leur investigation.

\section{Références}

Agache R., 1978. La Somme pré-romaine et romaine d'après les prospections à basse altitude. Mémoires de la Société des Antiquaires de Picardie, Amiens.

Antrop M., 1979. The interpretation of soil marks on aerial photographs recorded over the Famenne (Belgium) natural region. Pedologie XXIX, 209-240.

Aqdus S.A., Hanson W.S., Drummond J., 2007. Finding archaeological cropmarks: a hyperspectral approach, Proceedings of SPIE, the International Society for Optical Engineering. Presented at the Remote sensing for environmental monitoring, GIS applications, and geology, Society of Photo-Optical Instrumentation Engineers, 674908.1-674908.11. doi:10.1117/12.738007 pp. 
Aqdus S.A., Hanson W.S., Drummond J., 2012. The potential of hyperspectral and multi-spectral imagery to enhance archaeological cropmark detection: a comparative study, Journal of Archaeological Science, 39, pp. 1915-1924. doi:10.1016/j.jas.2012.01.034

Beresford M.W., St Joseph J.K.S., 1979. Medieval England: an aerial survey. Cambridge.

Bréart B., Nowicki F., Léva C., 1999. Archéologie aérienne. Actes du colloque international tenu à Amiens (France) du 15 au 18 octobre 1992. Hommage à Roger Agache pour 35 ans de prospections aériennes dans le Nord de la France, Revue Archéologique de la Picardie, $n^{\circ}$ spécial.

Brivio P.A., Pepe M., Tomasoni R., 2000. Multispectral and multiscale remote sensing data for archaeological prospecting in an alpine alluvial plain, Journal of Cultural Heritage, 1, pp. 155-164. doi:10.1016/S1296-2074(00)00155-2

Challis K., Kokalj Z., Kincey M., Moscrop D., Howard A.J., 2008. Airborne lidar and historic environment records. Antiquity, 82, pp. 1055-1064.

Devereux B.J., Crow G.S., Amable P., Cliff A.D., 2005. The potential of airborne lidar for detection of archaeological features under woodland canopies. Antiquity, 79, pp. 648-660.
Edis J., Mac'Leod D., Beawley R., 1989. An archaeologist's guide to classification of cropmarks and soilmarks. Antiquity, 63, pp. 112-126.

Lasaponara R., Masini N., 2006. Identification of archaeological buried remains based on the normalized difference vegetation index (NDVI) from Quickbird satellite data. IEEE Geosci. Remote Sens. Lett. 3, pp. 325-328. doi:10.1109/LGRS.2006.871747

Poirier N., Hautefeuille F., Calastrenc C., 2013. Low Altitude Thermal Survey by Means of an Automated Unmanned Aerial Vehicle for the Detection of Archaeological Buried Structures. Archaeological Prospection, 20, pp. 303-307. doi:10.1002/arp.1454

Trément F., 2000. L'apport des méthodes non-destructives à l'analyse des sites archéologiques: le point de vue de l'archéologue, in: Pasquinucci M., Trément F. (dir.), The Archaeology of Mediterranean Landscape: Non-Destructive Techniques Applied to Landscape Archaeology. Oxbow Books, Oxford, pp. 1-10.

Zadora-Rio É., 1991. Les terroirs médiévaux dans le Nord-ouest de l'Europe, in: Guilaine, J. (dir.), Pour Une Archéologie Agraire. Paris, pp. 165-192. 